\title{
Insecticide resistance and malaria transmission: infection rate and oocyst burden in Culex pipiens mosquitoes infected with Plasmodium relictum
}

\author{
Julien Vézilier ${ }^{1 *}$, Antoine Nicot', Sylvain Gandon², Ana Rivero ${ }^{1}$
}

\begin{abstract}
Background: The control of most vectors of malaria is threatened by the spread of insecticide resistance. One factor that has been hitherto largely overlooked is the potential effects of insecticide resistance on the ability of mosquitoes to transmit malaria: are insecticide-resistant mosquitoes as good vectors of Plasmodium as susceptible ones? The drastic physiological changes that accompany the evolution of insecticide resistance may indeed alter the ability of vectors to transmit diseases, a possibility that, if confirmed, could have major epidemiological consequences.
\end{abstract}

Methods: Using a novel experimental system consisting of the avian malaria parasite (Plasmodium relictum) and its natural vector (the mosquito Culex pipiens), two of the most common mechanisms of insecticide resistance (esterase overproduction and acetylcholinesterase modification) were investigated for their effect on mosquito infection rate and parasite burden. For this purpose two types of experiments were carried out using (i) insecticideresistant and susceptible laboratory isogenic lines of $C$. pipiens and (ii) wild $C x$. pipiens collected from a population where insecticide resistant and susceptible mosquitoes coexist in sympatry.

Results: The isogenic line and wild-caught mosquito experiments were highly consistent in showing no effect of either esterase overproduction or of acetylcholinesterase modification on either the infection rate or on the oocyst burden of mosquitoes. The only determinant of these traits was blood meal size, which was similar across the different insecticide resistant categories in both experiments.

Conclusions: Insecticide resistance was found to have no effect on Plasmodium development within the mosquito. This is the first time this question has been addressed using a natural mosquito-Plasmodium combination, while taking care to standardize the genetic background against which the insecticide resistance genes operate. Infection rate and oocyst burden are but two of the factors that determine the vectorial capacity of mosquitoes. Other key determinants of parasite transmission, such as mosquito longevity and behaviour, or the parasite's incubation time, need to be investigated before concluding on whether insecticide resistance influences the ability of mosquitoes to transmit malaria.

\section{Background}

Many of the most dangerous human diseases are transmitted by mosquitoes. Insecticide use is the mainstay of mosquito control programmes [1], and insecticide resistance one of its biggest obstacles [2]. Insecticide resistance jeopardizes disease control efforts by increasing the number of mosquitoes that survive the insecticide

\footnotetext{
* Correspondence: julien.vezilier@ird.fr

'Génétique et Evolution des Maladies Infectieuses (UMR CNRS 2724), Centre de Recherche IRD, 911 Avenue Agropolis, 34394 Montpellier, France Full list of author information is available at the end of the article
}

treatment and are available to spread the parasite in the population. Although this quantitative effect of insecticide resistance on the mosquitoes may be mitigated by the costs associated to insecticide resistance [3-6] it has been deemed sufficiently worrying to motivate the development of resistance management strategies to prevent or retard the spread of resistance [7-9]. Little attention has however been given to the potential qualitative effects of insecticide resistance on the mosquitoes: are insecticide resistant mosquitoes better, equal or worse vectors of diseases than susceptible ones? 
Mosquitoes are not mere flying syringes, they provide a very specific physiological environment in which parasites differentiate, proliferate and migrate to the correct tissues to ensure transmission to the next host. There is increasing evidence that this environment is drastically modified when insects become resistant to insecticides [10]. For instance, recent work by McCarroll and coworkers $[11,12]$ has shown that insecticide resistance levels in wild Culex quinquefasciatus mosquitoes are negatively correlated with the density of the filarial parasite Wuchereria bancrofti, and that parasite development is blocked at the L1 stage in laboratory mosquitoes selected for artificially high levels of insecticide resistance $[11,13])$. These results could extend to other mosquito-parasite combinations and have large implications for the transmission of diseases.

Malaria is one of the most lethal diseases of humans. It is caused by protozoa of the genus Plasmodium that parasitize mammals, birds and lizards. Mosquito control through the use of insecticides is still the most important component of malaria control programmes. Insecticide resistance, including multiple resistance to all the major classes of insecticides, has been reported in all the main mosquito vectors of malaria [14]. Mosquitoes are able to escape the lethal effects of insecticides by two non exclusive physiological strategies: by reducing the sensitivity of the neural targets of the insecticides (target site resistance) and by increasing the activity of detoxifying enzymes (metabolic resistance [2]). In some areas, the prevalence of these types of insecticide resistance is so high - upwards of $50 \%[15,16]$ - that Plasmodium parasites circulating in the blood of infected hosts have a high chance of being ingested by an insecticide resistant mosquito. Despite this, surprisingly little is known as to whether insecticide resistance interferes with the subsequent development of Plasmodium within the vector. This important gap in our knowledge is partly due to the difficulties associated with finding sympatric insecticide-resistant and susceptible mosquitoes, particularly in areas with a long and complex history of insecticide use where multiple resistance mechanisms are the norm [17] and fully susceptible individuals hard to find. This has driven researchers to work on allopatric mosquito combinations $[18,19]$ or on mosquito-Plasmodium combinations not found in nature [20], both of which render the results difficult to interpret [10].

This study investigates the potential effect of insecticide resistance on Plasmodium infection rate and parasite burden within mosquitoes using the avian malaria parasite, Plasmodium relictum, and its natural mosquito vector, Culex pipiens [21]. Avian malaria parasites share a distant common ancestor with human malaria parasites [22] and have historically played an important role as models in the study of human malaria [23]. Culex pipiens is one of the main vectors of avian Plasmodium [21] and is widely present in the south of France, where it is seen as a nuisance to the tourist industry. Following repeated treatments of larval sites with organophosphate insecticides (initiated 40 years ago), the two main types of insecticide resistance are present in the Montpellier region: target site resistance (through the modification of the acetylcholinesterase [24]) and metabolic resistance (through the overproduction of detoxifying carboxylesterases [25]). One particularly convenient feature of this system is that mosquito control has been limited to the populations along the Mediterranean coast. It is thus possible to identify an insecticide-treated area (a $20 \mathrm{Km}$ band close to the sea), a non-treated area (further north), and an intermediate area where metabolic and target site resistant mosquitoes coexist with susceptible ones [26]. In addition, through a series of back-crossings carried out at the Institut des Sciences de l'Evolution de Montpellier, the different insecticide resistance alleles found in the region have been separately introgressed into a common (insecticide-susceptible) genetic background to produce different isogenic insecticide-resistant mosquito lines [4].

Qualitatively different predictions can be made on the effect of the two different insecticide resistant mechanisms on Plasmodium development within the mosquito. These have been extensively reviewed elsewhere [10] and will only be briefly outlined here. First, the overproduction of large amounts of detoxifying esterases results in a substantial depletion of the energetic stores of mosquitoes [27]. Resource depletion can have two contrasting consequences for parasite development: on the one hand it may hinder mosquito immunity, whose maintenance and deployment are known to be resource dependent [28], thereby favoring parasite development. On the other hand, it may limit the development of Plasmodium, a parasite whose mosquito stages are known to be greedy consumers of resources [29,30]. Second, esterases have been shown to be highly expressed in mosquito tissues that are key for parasite development (such as the midgut [31]). These overproduced esterases may render these tissues toxic for parasite development through, amongst others, an excess production of reactive oxygen species - a possibility that has never been formally explored but for which there is indirect evidence $[10,11]$. Finally, acetylcholinesterase modification has been shown to increase the feeding rate of $C x$. pipiens larvae [32], most likely as a consequence of an hyperactive nervous system resulting from an excess of acetylcholinesterase in the synapses [3]. Hyperactivity may also have an impact on the blood feeding efficiency 
of the adults and thus on the number of parasites ingested, though not necessarily on their subsequent development [10].

This paper aims to reply to the following three questions: 1) Do insecticide resistant mosquitoes have a different probability of infection than susceptible ones? 2) Do infected insecticide resistant mosquitoes attain higher or lower parasite burdens than susceptible ones? and 3) Are these differences dependent on the underlying insecticide resistance mechanism (metabolic vs target site)? For this purpose, experimental P. relictum infections were carried out using (i) insecticide-resistant and susceptible laboratory isogenic lines of $C x$. pipiens and (ii) wild $C x$. pipiens collected from a population where insecticide resistant and susceptible mosquitoes coexist in sympatry. The isogenic lines allowed us to test the effect of the insecticide resistance genes in a uniform genetic background. This may increase the chances of detecting an eventual pleiotropic effect of the insecticide resistance genes, but the results may not be necessarily applicable to other genetic backgrounds, particularly if there are epistatic interactions between the insecticide resistant genes and other genes in the genome (although modifier genes have not yet been described in this species [33]). The field-collected mosquitoes, on the other hand, allowed us to test the effects under the more realistic conditions of a heterogeneous genetic background [34]. Combined, these two approaches provide a powerful test of the role of insecticide resistance on parasite development within the mosquito.

\section{Methods}

Avian malaria parasite

Plasmodium relictum (lineage SGS1) is the aetiological agent of the most prevalent form of avian malaria in Europe [21]. This generalist Plasmodium parasite lineage was originally isolated by G. Sorci (CNRS, Dijon) from wild sparrows caught in the region of Dijon (France) in 2008 (wild mosquito experiments) and 2009 (isogenic line experiments) and subsequently passaged to naïve canaries (Serinus canaria) by intra peritoneal injection.
The strain was maintained in an animal house by carrying out regular passages between stock canaries every ca. 3 weeks. At the time of the experiments, the parasite had undergone between nine (wild mosquito experiments) and fourteen (isogenic line experiments) passages since their transfer from the sparrows. For the purpose of the experiments, the experimental canaries were infected by intra-peritoneal injection of ca. 50-100 $\mu \mathrm{L}$ of blood from the infected canary stock. Their parasitaemia was regularly monitored from the fifth day of infection onwards using thin blood smears as described in [21]. Mosquito feeding (see below) took place 10 days after the onset of the infection, to coincide with the acute phase of the parasitaemia (Vézilier, unpublished results).

\section{Isogenic strain experiments \\ Mosquito rearing}

Experiments were carried out using one insecticide susceptible strain (SLAB), two insecticide resistant strains through the overproduction of detoxifying esterases (SA2B2, SA4B4) and one strain with an insensitive acetylcholinesterase but no overproduced esterases (SR). Details of these strains are given in Table 1. Eggs of each of the different mosquito strains were obtained from the Institut des Sciences de l'Evolution de Montpellier and set up to hatch under standard insectary conditions $\left(25 \pm 1^{\circ} \mathrm{C}, 70 \pm 5 \% \mathrm{RH}\right.$ and $12 \mathrm{~L}$ : $12 \mathrm{D}$ photoperiod). On the hatching day, larvae were haphazardly seeded into plastic trays (4 trays per genotype, dimensions: $25 \mathrm{~cm} \times 35 \mathrm{~cm} \times 7 \mathrm{~cm})$ containing one litre of mineral water (Eau de Source Carrefour, France) at a constant density of 300 individuals per tray. Larvae were provided with a half-tablet of concentrated yeast on the day of the hatching, $200 \mathrm{mg}$ of TetraMin ${ }^{\circledR}$ fish flakes the following day, and from then on $400 \mathrm{mg}$ TetraMin every two days until pupation. Tray water was changed on feeding days to avoid bacterial growth on the water surface. On pupation, trays were placed inside an emergence cage $(27 \times 40 \times 35 \mathrm{~cm})$ and provided with an $a d$ libitum source of $10 \%$ sugar solution for the emerged adults.

Table 1 Insecticide resistant and susceptible strains used in the isogenic strain experiment

\begin{tabular}{|c|c|c|c|}
\hline Strain & IR mechanism & Alleles & Genetic background \\
\hline SLAB & None & Ester $^{0}$, ace $^{-1} 1^{\mathrm{s}}$ & SLAB \\
\hline SA2B2 & Overproduction of esterases A2 and B2 & Ester $^{2}$, ace $^{-1}{ }^{\mathrm{S}}$ & SLAB \\
\hline SR & Insensitive acetylcholinesterase & Ester $^{0}$, ace $^{-1} 1^{R}$ & SLAB \\
\hline
\end{tabular}

The overproduction of esterases is controlled by a superlocus consisting of two loci (esterase $A$ and esterase $B$ ) in complete linkage disequilibrium. Alleles for this locus are the wild type susceptible Ester ${ }^{0}$, or the insecticide resistant Ester ${ }^{2}$ (overproduces the esterase A2 and B2 isozymes) and Ester ${ }^{4}$ (overproduces the esterase A4 and B4 isozymes). The modification of the acetylcholinesterase is controlled by the locus ace- 1 . Alleles for this locus are the wild type susceptible ace- $1^{S}$ and the insecticide resistant $a c e-1^{R}$ (which contains a single GGC $\rightarrow$ AGC point mutation that renders the acetylcholinesterase insensitive to the insecticide). For more details on those strains, see [4]. 


\section{Mosquito experimental infections and dissections}

One day before the feeding, 50 female mosquitoes from each of the four strains (SLAB, SA4B4, SA2B2, SR) were haphazardly chosen from the different emergence cages and placed inside an experimental cage. Three such experimental cages were obtained in this way. The mosquitoes in these experimental cages were deprived of glucose for $24 \mathrm{~h}$ to increase hunger levels and thus favour blood feeding. On the morning of the blood feeding day, bird parasitaemia was estimated by counting the proportion of infected red blood cells using Giemsa-stained blood smears. Blood feeding was carried out overnight by placing a different experimental Plasmodium-infected canary inside an upturned aerated containment box (diameter $14 \mathrm{~cm}$, height $12 \mathrm{~cm}$ ) on top of each cage. The canaries stood on top of the cage allowing the mosquitoes to feed on the bird's feet through the screened wall, while the rest of the body was protected from the bites. Engorged females were taken out from the cages, briefly anesthetized with $\mathrm{CO}_{2}$ and placed individually into numbered dry 30 $\mathrm{ml}$ drosophila plastic tubes covered with a mesh. Food was provided in the form of a cotton pad soaked in a $10 \%$ glucose solution placed on top of each tube. This cotton pad was replaced daily throughout the remainder of the experiment.

Due to the high number of replications and the time required to dissect out and count oocysts in the mosquito gut, mosquito dissections were spread out over four consecutive days: days 5-8 post blood meal (pbm). Each dissection day, one fourth of the blood-fed mosquitoes that had fed on each of the canaries were haphazardly chosen, taken out from their tubes and dissected under a binocular microscope in $100 \mu \mathrm{l}$ of $0.01 \mathrm{M}$ phosphate-buffered saline (PBS). The tubes were kept at $4^{\circ} \mathrm{C}$ for haematin analysis (see below). The dissected midguts were transferred with a pin to a slide containing a drop of PBS with $5 \%$ mercurochrome. The slide was observed under a phase contrast microscope equipped with a 40x oil immersion objective to assess infection rate (oocysts present/absent) and oocyst burden (number of oocysts in infected guts). Dissected bodies were kept on ice immediately after dissection and subsequently frozen at $-80^{\circ} \mathrm{C}$ for genotype identification. Mosquito genotype (SLAB, SA2B2, SA4B4 or SR) was determined using RFLP analysis as described in [35,36]. Quantification of the haematin (a by-product of the decomposition of haemoglobin) excreted at the bottom of the tubes was carried out as described in previous papers [37]. The purpose of haematin quantification is to correct for potential differences in the amount of blood ingested by females of different genotypes. Solutions with an absorbance $\leq 0.01$ were classified as being from mosquitoes that had not blood fed, as this absorbance was indistinguishable from the $\mathrm{LiCO}_{3}$ control.

\section{Wild mosquito experiments}

\section{Mosquito collections and rearing}

Wild Cx. pipiens mosquito larvae were collected in August (Block 1), September (Block 2) and October (Block 3) 2008 from a sympatric population of insecticide resistant and susceptible mosquitoes. This population was found $20 \mathrm{~km}$ north of Montpellier, in a sewage treatment lagoon (near the village of Triadou, France) located at the boundary between an insecticide-treated and non-treated zone [33]. A previous sampling of the larvae in this basin carried out in June of the same year had given a balanced proportion of each of the different insecticide resistant genotypes (data not shown). The larvae collected were brought to the insectary, sorted by developmental stage, and seeded haphazardly in five plastic trays at a constant density of 300 individuals per tray. The rearing conditions were identical to those used for the isogenic strains (see above).

Two additional trays were seeded with 300 larvae of the SLAB laboratory strain of Cx. pipiens (see above). The SLAB mosquitoes were reared in parallel and in identical (density, food, insectary) conditions to the wild collected mosquitoes. To discriminate SLAB from wild mosquitoes, four days before the experiment, SLAB females were marked with a RadGlo ${ }^{\circledR}$ JST fluorescent pigment applied using a dust storm technique as described in [38]. The amount of dust applied was $50 \mu \mathrm{g}$ for 50 individuals, which in preliminary trials was found to have no effect on mosquito survival or oocyst count (Flore Zélé and Julien Vézilier, unpublished data), and was only detectable using a binocular microscope.

\section{Mosquito experimental infection and dissections}

Experimental infections were carried out in identical way to the isogenic strain experiments (see above). Each cage (3 cages per block) contained 150 wild female mosquitoes and 50 marked SLAB mosquitoes, haphazardly chosen from the emergence cages. A different infected canary was placed overnight on top of each of the cages for the blood feeding to take place. The presence of SLAB mosquitoes in the cages served a double purpose: on the one hand, to obtain a standard measure of parasite infectivity to mosquitoes in each of the cages. On the other hand it allowed us to compare the infectivity of $P$. relictum in wild-caught vs. lab-reared isogenic lines.

As above, mosquito dissections were spread out over 4 consecutive days: days 5-8 post blood meal (pbm). Each day, one fourth of SLAB and wild mosquitoes ( $n=12$ SLAB and $n=25$ wild mosquitoes) from each cage were haphazardly chosen, taken out from their tubes and the measurements of infection rate, oocyst burden, and haematin quantification obtained as for the isogenic strain 
mosquitoes. Wild mosquitoes were typed to determine their insecticide status in the following ways. Insecticide resistance through modification of the acetylcholinesterase was established by analysing homogenates of the heads of individual mosquitoes using the micro-plate TPP test [39]. Insecticide resistance through the overproduction of carboxylesterases was analysed on single mosquito thorax homogenates by starch-gel electrophoresis in TME 7.4 buffer systems [40]. This technique allows us to distinguish between the three main different insecticide resistant carboxylesterase allozymes present in the study area: A1 (allele Ester ${ }^{1}$ ), A4-B4 (allele Ester ${ }^{4}$ ) and A2-B2 (allele Ester ${ }^{2}$ )[25]. Because of haemolymph loss (and subsequent active esterase loss) during the dissection process, some thorax homogenates didn't allow us to establish the carboxylesterase resistance status through starch-gel electrophoresis. In these cases ( $\mathrm{n}=$ 176), the abdomen homogenates were analysed using RFLP analysis (as above). Once typed, field collected mosquitoes were allocated to one of 4 insecticide resistance status: S (fully susceptible), E (resistant through esterase overproduction), A (resistant through acetylcholinesterase modification) or AE (resistant through both acetylcholinesterase modification and esterase overproduction, see Table 2 for details).

All experiments described above were conducted in accordance with the French Government regulations for animal experimentation. Canaries were housed in a licensed animal house (Direction Départementale des Services Vétérinaires de l'Hérault - DDSV, licence number: E 34-172-21). A. Rivero is authorized under French law to experiment on canaries (DDSV authorization number: 34.363).

Table 2 Number of mosquitoes from the different insecticide resistant categories used in the wild mosquito experiments.

\begin{tabular}{|c|c|c|c|c|}
\hline IR status & Alleles & Block 1 & Block 2 & Block 3 \\
\hline $\mathrm{S}$ & Ester $^{0}$, ace-1 ${ }^{\mathrm{S}}$ & $83(30.07 \%)$ & $88(27.16 \%)$ & $90(27.78 \%)$ \\
\hline \multirow[t]{3}{*}{ E } & Ester $^{1}$, ace-1 ${ }^{\text {s }}$ & $2(0.72 \%)$ & $7(2.16 \%)$ & $4(1.23 \%)$ \\
\hline & Ester $^{2}$, ace-1 ${ }^{\text {s }}$ & $7(2.54 \%)$ & $3(0.93 \%)$ & $2(0.62 \%)$ \\
\hline & Ester $^{4}$, ace-1 ${ }^{\text {s }}$ & 77 (27.9\%) & $98(30.25 \%)$ & $86(26.55 \%)$ \\
\hline A & Ester $^{0}$, ace $-1^{\mathrm{R}}$ & 42 (15.22\%) & $54(16.67 \%)$ & $62(19.13 \%)$ \\
\hline \multirow[t]{3}{*}{$A E$} & Ester $^{1}$, ace- $1^{R}$ & $2(0.72 \%)$ & $3(0.92 \%)$ & $6(1.85 \%)$ \\
\hline & Ester $^{2}$, ace-1 ${ }^{\mathrm{R}}$ & $4(1.45 \%)$ & $2(0.62 \%)$ & $3(0.93 \%)$ \\
\hline & Ester $^{4}$, ace $^{-1}{ }^{\mathrm{R}}$ & $59(21.38 \%)$ & 69 (21.29\%) & 71 (21.91\%) \\
\hline
\end{tabular}

Total $276(100 \%) \quad 324(100 \%) \quad 324(100 \%)$

The corresponding proportions are given in brackets. The different insecticide resistance status are S: fully susceptible, E: resistance through esterase

overproduction, A: resistant through acetylcholinesterase modification and AE: resistant through both esterase overproduction and AChE modification.

\section{Statistical analyses}

The analyses were carried out using the $\mathrm{R}$ statistical package (v.2.10.1). The different statistical models applied to the data are described in Additional File 1. The analysis of infection rate (oocyst presence/absence) was carried out using the lmer mixed model procedure with binomial errors (lme4 package). For the analysis of infection intensity (number of oocysts), only individuals that developed $\geq 1$ oocyst were included. As has been found in other systems $[41,42]$ oocyst count data were greatly overdispersed. One way of handling this overdispersion is by using negative binomial pseudo distributions [42]. However, to date, it is not currently possible to account for negative binomial distributions within a mixed model lmer procedure. For this reason, data were Box-Cox transformed and analysed using the lme mixed model procedure (nlme package). For the graphical analysis of the relationship between oocyst burden and haematin (individuals that developed $\geq 1$ oocyst), the gam procedure was used (mgcv package).

The insecticide resistance status of the insects (SLAB, SA4B4, SA2B2, SR in the isogenic strain experiment and $\mathrm{S}, \mathrm{E}, \mathrm{A}, \mathrm{AE}$ in the wild mosquito experiments), and the amount of haematin (the standard proxy for blood meal size [43]) were fitted as fixed explanatory variables. Maximal models were simplified by sequentially eliminating non-significant terms and interactions to obtain a minimal model [44]. The significance of the explanatory variables was established using a likelihood ratio test (LRT) which is approximately distributed as a $\chi^{2}$ distribution [45]. The significant $\chi^{2}$ values given in the text are for the minimal model, while non-significant values correspond to those obtained before deletion of the variable from the model. When appropriate, such as in the analysis of the significant effect of insecticide resistance status in block 3 of the wild mosquito experiments, $a$ posteriori contrasts were carried out by aggregating factor levels that did not significantly differ from each other and by testing the fit of the simplified model using an LRT [44]. As oocyst burden could be expected to be a non-linear function of haematin quantity, the quadratic term haematin ${ }^{2}$ was added to the minimal model to assess if it significantly improved the model fit. Differences between birds and dissection days were often significant but as they are of no interest in their own right, they were controlled for by leaving these terms as random factors in the model (see Additional File 1). The one exception was in the comparison of SLAB and wild-caught mosquitoes (models 19 and 20) where dissection day had to be fitted as a fixed factor rather than a random factor, as the latter did not allow for model convergence (dissection day was however not retained in the minimal model). The three blocks of the wild mosquito experiments, which were obtained at 
three different monthly intervals, were first analysed separately (one analysis per block). A second, more stringent, test was subsequently carried out by analyzing all blocks together and adding block as an additional random factor to the model.

\section{Results}

\section{Isogenic strain experiments}

After overnight exposure with the infected canaries, $85 \%$ of the SLAB, $88 \%$ of the SA4B4, $94 \%$ of the SA2B2 and $85 \%$ of the SR mosquitoes had taken a blood meal and were subsequently dissected for oocyst detection. The rest either died before feeding or did not blood feed.

There were no significant differences between the insecticide resistant and susceptible strains in either blood meal size (model $1, \chi^{2}{ }_{3}=1.56, \mathrm{p}=0.66$ ), probability of infection (model $2, \chi_{3}^{2}=1.70, p=0.63$, Figure 1a) or oocyst burden (model $3, \chi_{3}^{2}=6.90, \mathrm{p}=0.08$, Figure 2a). The probability of becoming infected with $P$. relictum and the amount of oocysts that successfully developed in the mosquito increased drastically with the amount of blood consumed (model $2, \chi^{2}{ }_{1}=97.5, \mathrm{p}<$ 0.0001 and model $3, \chi^{2}{ }_{1}=112.55, \mathrm{p}<0.0001$, respectively), irrespective of the strain. Fitting the quadratic term (haematin ${ }^{2}$ ) highly improved the model fit (model $3, \chi_{1}^{2}=24.95, \mathrm{p}<0.0001$ ), suggesting that oocyst burden was a decelerating polynomial function of blood meal size (Figure 3a).

\section{Wild mosquito experiments}

The number of field-collected mosquitoes from each allelic variant that took a blood meal and were subsequently dissected for oocyst detection is given in Table 2. The oocystaemias observed in the wild mosquito experiment were, on average, significantly larger than those found in the isogenic strain experiment (mean \pm s.e., $208 \pm 10$ and $16 \pm 1$ respectively, all strains combined, $\chi_{1}^{2}=5.46, \mathrm{p}=$ 0.02 ). This turned out to be an experiment effect, rather than a laboratory vs field mosquito effect, as the comparison of SLAB vs field-caught mosquitoes within the wild mosquito experiment showed no significant differences in either infection probability (model 19: $\chi^{2}{ }_{1}=0.0084$, $\mathrm{p}=0.93$, Figure $1 \mathrm{~b}-\mathrm{d}$ ), or oocyst burden (model 20: $\chi^{2}{ }_{1}=$ $1.93, \mathrm{p}=0.16$, Figure $2 \mathrm{~b}-\mathrm{d}$ ).

The effect of insecticide resistance status on blood meal size, infection probability and oocyst burden was first analysed separately for each block. For the first two blocks, blood meal size was independent of the insecticide resistant status of the mosquitoes (Block 1 , model $4: \chi^{2}{ }_{3}=4.10, \mathrm{p}=0.25$; Block 2 , model 8: $\chi^{2}{ }_{3}=4.31, \mathrm{p}=$ $0.23)$. In block 3 , however, $\mathrm{S}$ females ingested slightly more blood than the other insecticide resistant categories (model 12, $\chi_{3}^{2}=9.54, p=0.023$ ). In all three blocks the infection rate was strongly dependent on the amount of haematin ingested (Block 1, model 5: $\chi^{2}{ }_{1}=$ 13.14, $\mathrm{p}=0.0003$; Block 2, model 9: $\chi^{2}{ }_{1}=33.38, \mathrm{p}<$ 0.0001; Block 3, model 13: $\chi^{2}{ }_{1}=8.13, \mathrm{p}=0.0043$ ) but independent of the insecticide resistance status of the mosquitoes (Block 1, model 5: $\chi^{2}{ }_{3}=4.11, \mathrm{p}=0.25$; Block 2, model 9: $\chi_{3}^{2}=1.73, \mathrm{p}=0.63$; Block 3, model 13: $\chi_{3}^{2}=1.44, \mathrm{p}=0.69$, Figure $\left.1 \mathrm{~b}-\mathrm{d}\right)$. Oocyst burden was also strongly positively correlated to the amount of haematin ingested (Block 1, model 6: $\chi^{2}{ }_{1}=74.57, \mathrm{p}<$ 0.0001; Block 2, model 10: $\chi^{2}{ }_{1}=27.89, \mathrm{p}<0.0001$; Block 3, model 14: $\left.\chi^{2}{ }_{1}=49.00, \mathrm{p}<0.0001\right)$. Adding the quadratic term (haematin ${ }^{2}$ ) significantly improved the model fit in two blocks out of three (Block 1, model 6: $\chi^{2}{ }_{1}=6.30, \mathrm{p}=0.01$; Block 2, model 10: $\chi^{2}{ }_{1}=8.79, \mathrm{p}=$ 0.003 , Block 3, model 14: $\chi^{2}{ }_{1}=2.58, \mathrm{p}=0.11$ ). The insecticide resistance status of mosquitoes was not a significant explanatory factor of oocyst burden in blocks 1 (model 6, $\chi_{3}^{2}=3.85, \mathrm{p}=0.28$, Figure $2 \mathrm{~b}$ ) and 2 (model $10, \chi_{3}^{2}=1.01, \mathrm{p}=0.80$, Figure $2 \mathrm{c}$ ), but became significant in block 3 (model $14, \chi^{2}{ }_{3}=10.50, p=0.01$, Figure $2 \mathrm{~d})$. In this block, insecticide resistant mosquitoes had significantly fewer oocysts than the susceptible ones (Block 3, model 14: $\chi^{2}{ }_{1}=7.49, \mathrm{p}=0.006$ ), independently of the insecticide resistance mechanism involved (Block 3, model 14: $\chi_{2}^{2}=3.02, \mathrm{p}=0.22$ ).

Analysing the three blocks together did not alter the results for infection rate, which was still independent of insecticide resistance status (model 16: $\chi_{3}^{2}=0.79, \mathrm{p}=$ 0.85 ). The significant effect of insecticide resistance on oocyst burden, however, disappeared (model 17: $\chi^{2}{ }_{3}=$ $2.76, \mathrm{p}=0.43$ ), leaving only haematin and haematin ${ }^{2}$ as explanatory variables in the model (Figure $3 \mathrm{~b}$ ). Using the mean number of oocysts in SLAB mosquitoes as a standard measure of parasite infectivity did not alter any of the above results (models 7, 11, 15 and 18).

\section{Discussion}

An extensive investigation into the effect of two main mechanisms of insecticide resistance on the infection rate and parasite burden of $P$. relictum infections was carried out by means of a series of experiments using both isogenic mosquito lines and field-collected mosquitoes. Infection rate determines the proportion of infected mosquitoes in a population and is a key parameter in models of malaria transmission [46]. Oocyst burden is directly related to the number of transmissible (sporozoites) stages that subsequently develop in the mosquito salivary glands [41] and has been recently shown to be strongly correlated with mosquito longevity, a key component of the mosquito's vectorial capacity [47].

It has been suggested that different mechanisms of insecticide resistance could alter both of these parameters [10], and similar insecticide resistant mechanisms have been shown to alter the development of other 

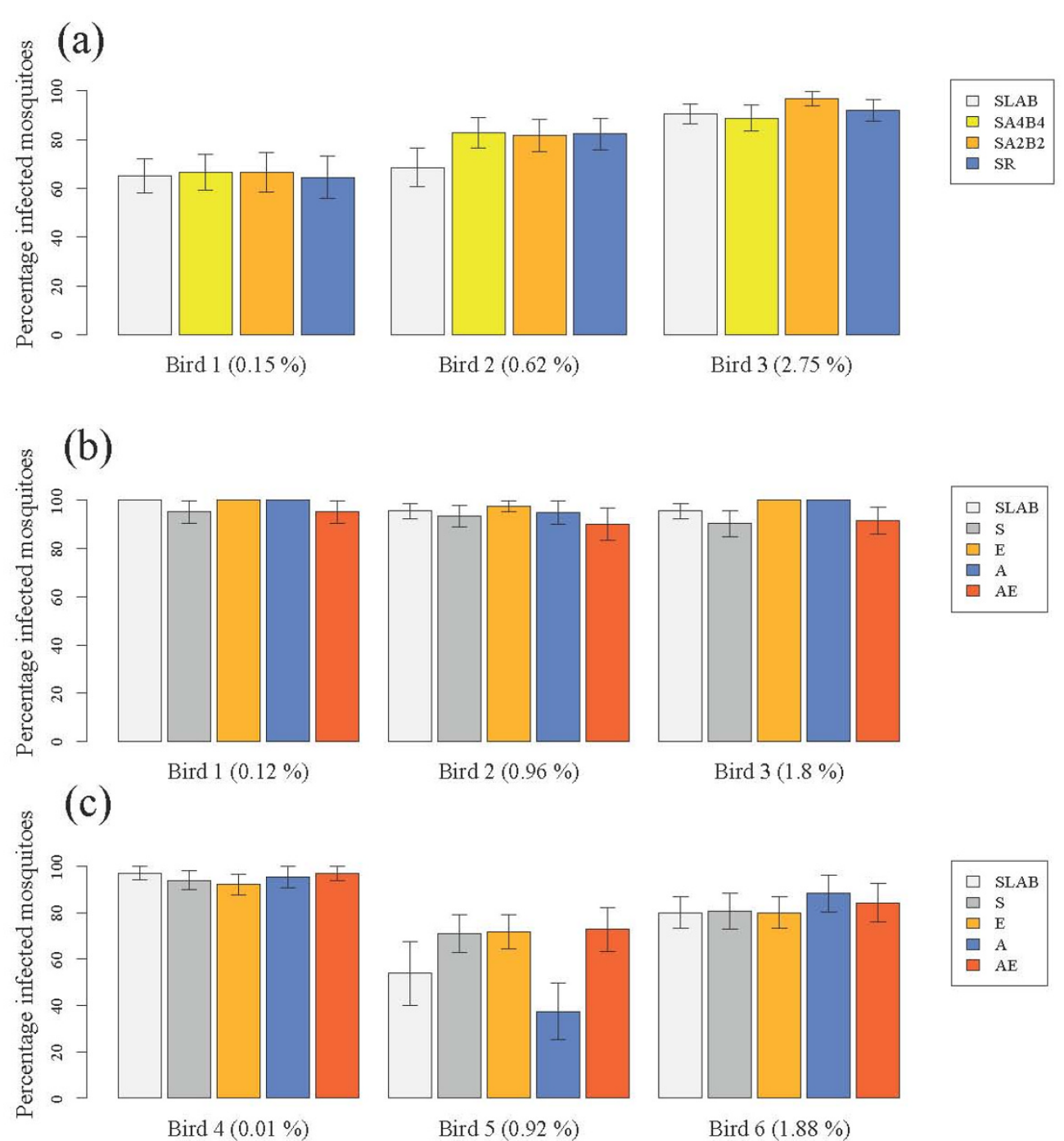

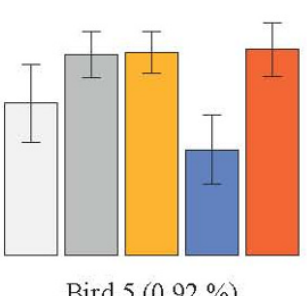

Bird $5(0.92 \%)$

(d)

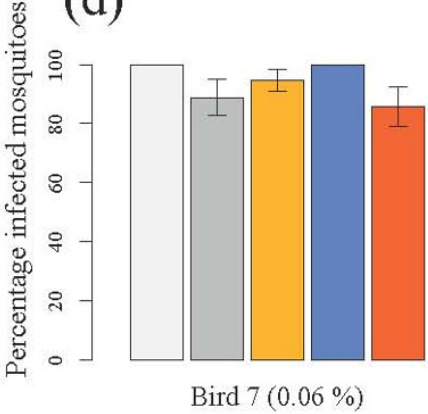

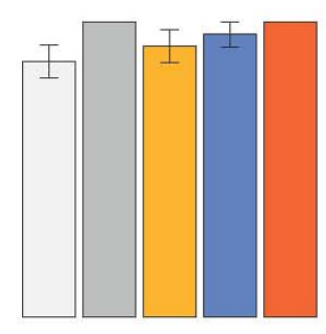

Bird $8(0.42 \%)$

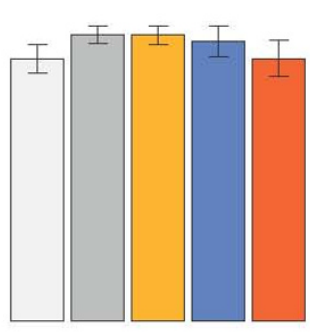

Bird $9(6 \%)$ $\square$ SLAB

$\square \mathrm{S}$

$\square \mathrm{E}$

$\square \mathrm{A}$

Figure 1 Infection rate of insecticide-resistant and susceptible mosquitoes in (a) the isogenic strain experiment and (b) block 1 , (c) block 2 and (d) block 3 of the wild mosquito experiments. Three different experimentally infected birds were used in each of the four experiments (bird parasitaemia at the day of the feed is indicated in brackets). The figure shows the mean ( \pm se) proportion of mosquitoes with at least one oocyst in. See Tables 1 and 2 for details of mosquitoes used in each experiment. 
$-$
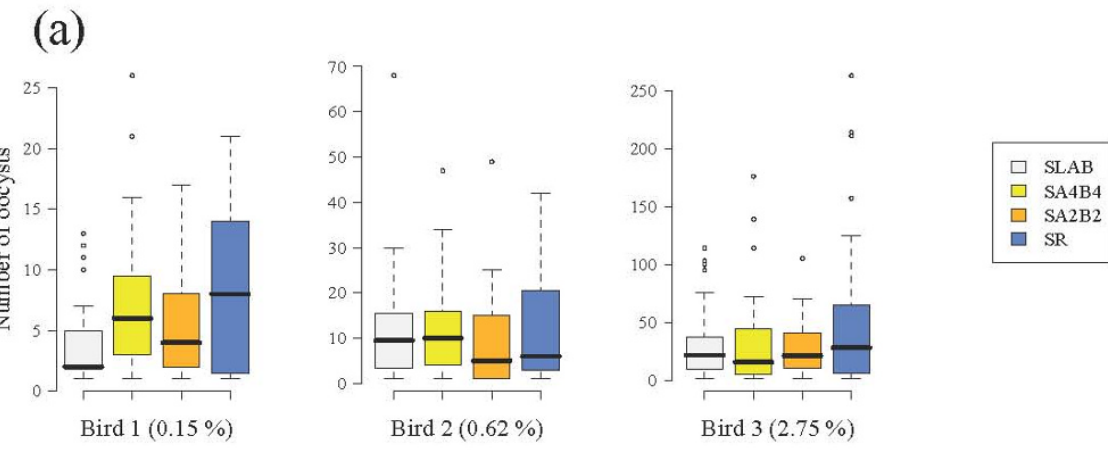

(b)
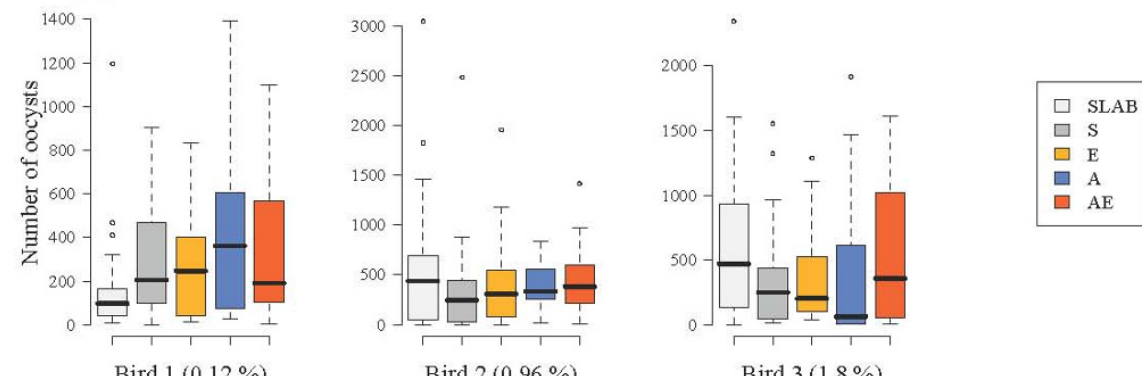

(c)
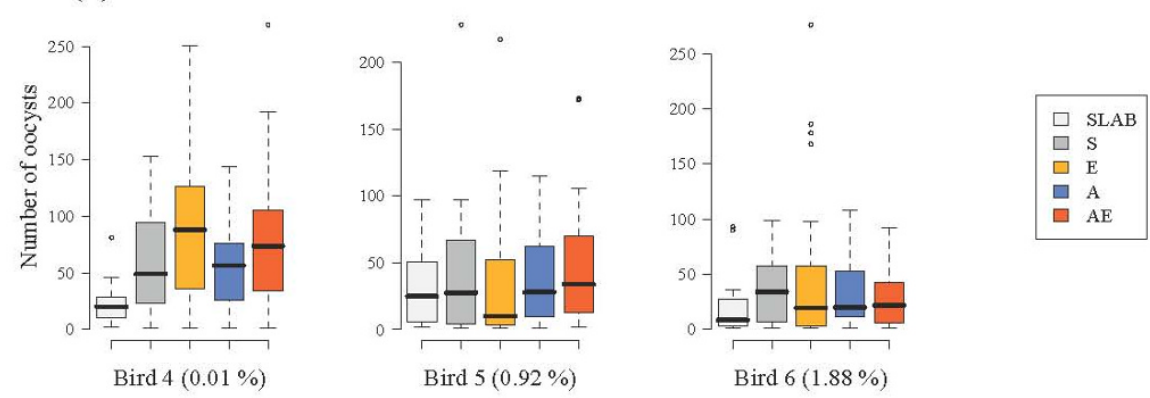

(d)
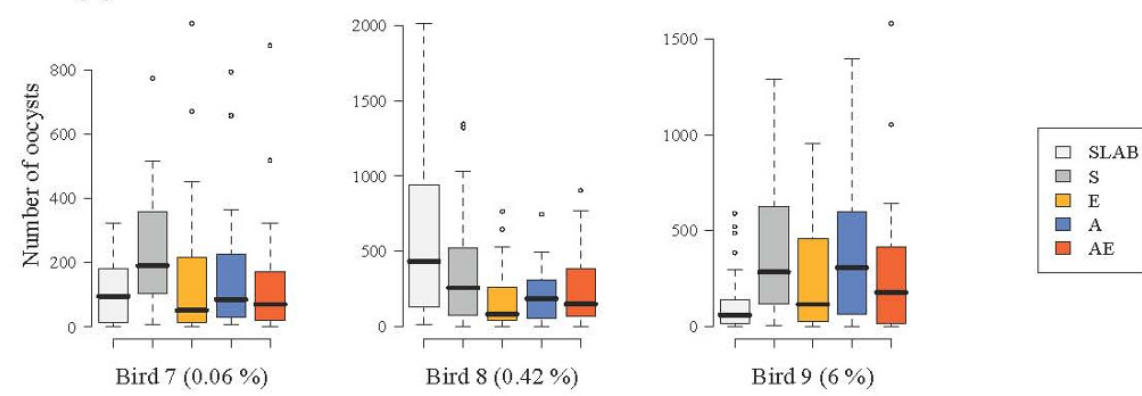

Figure 2 Oocyst burden of insecticide resistant and susceptible mosquitoes in (a) the isogenic strain experiment and (b) block 1 , (c) block 2 and (d) block 3 of the wild mosquito experiments. Three different experimentally infected birds were used in each of the four experiments (bird parasitaemia at the day of the feed is indicated in brackets). The figure shows the median number of oocysts (horizontal black bars). The coloured boxes below and above the median indicate the first and third quartiles respectively. Dashed lines delimit 1.5 times the inter-quartile range on both side of the box, above which individual counts are considered outliers and marked as dots. See Tables 1 and 2 for details of mosquitoes used in each experiment. 
(a)

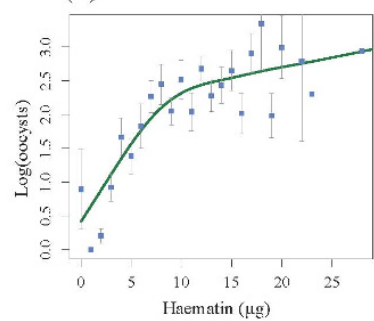

(b)

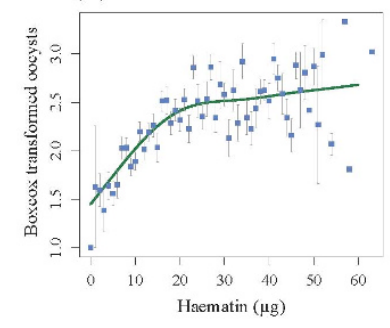

Figure 3 Relationship between number of oocysts and blood meal size in (a) the isogenic strain experiment and (b) cumulative dataset for the three wild mosquito experiments General additive models were fitted to each dataset using all mosquito replicates independently of their insecticide resistant status. Squares represent the mean number of oocysts for each haematin value (haematin values were rounded up to the nearest integer). Bars above and below the means represent the standard errors of the mean. The fitted curve corresponds to the predicted values arising from the statistical models.

parasites $[11,12]$. However, aside from a marginal, albeit statistically significant, reduction in oocyst burden in insecticide resistant insects within the third block of the wild mosquito experiments, no clear effect of either esterase overproduction or of acetylcholinesterase modification on either infection rate (proportion of mosquitoes containing at least one oocyst) or oocyst burden was found. The isogenic line and wild-caught mosquito experiments were consistent in showing that the only determinant of infection rate and oocyst burden was blood meal size, which was similar across the different insecticide resistant categories in both experiments. The number of oocysts in the midgut of infected mosquitoes was found to be a saturating function of blood meal size: as the amount of blood ingested increases, the number of ookinetes that successfully encyst in the mosquito midgut reaches a limit. Provided that blood meal size was directly proportional to the amount of parasites ingested, these results seem to be in accordance with a recent study showing the existence of a saturating gametocyte-ookinete and/or ookinete - oocyst transition associated to high parasite densities [41].

There are two potential explanations for the lack of insecticide resistance effects on infection rate and oocyst burden. The first explanation is that, contrary to predictions, the physiological alterations associated to insecticide resistance are not sufficiently important to alter the development of $P$. relictum within the mosquito. Vontas et al [20] also failed to show differences in parasite burden when comparing a pyrethroid-resistant and a susceptible strain of Anopheles stephensi infected by Plasmodium yoelii. Their results were however difficult to interpret for two reasons. Firstly, because the insecticide resistant and susceptible strains had different geographic origins (the resistant DUB-R strain originated from Dubai in 1986, while the susceptible BEECH strain originated from India in the 1940s). The genetic background differences that inevitably arise during such a divergent evolutionary history could have potentially blurred the pleiotropic effects of insecticide resistant genes on Plasmodium development. Secondly, because $A n$. stephensi is not a natural vector for P. yoelii. Lessons learned from immune [48,49] and longevity [50] studies, have taught us that natural and unnatural mosquito-Plasmodium combinations can render substantially different results (see also $[34,51,52]$ ). The present study overcomes this two potential pitfalls by using a natural vector-mosquito combination [21] and by carrying the experiments using both isogenic mosquito strains and sympatric wild-caught mosquitoes.

The second potential explanation is that the high oocystaemias obtained in the experiments may have swamped any eventual physiological differences existent between insecticide resistant and susceptible mosquitoes. Oocystaemias within the range of those found in this study are a common outcome when human malaria vectors (Anopheles $s p$ ) are experimentally infected with rodent malaria parasites $[41,47,53]$ and contrast sharply with the low oocystaemias found in Anopheles mosquitoes caught in endemic human malaria areas [51]. Part of the reason may lie in the novel nature of such experimental host-parasite associations, which can result in increased parasite virulence $[34,51]$. To date, no studies have investigated $P$. relictum oocystaemias in wildcaught mosquitoes, but it is safe to assume that, despite being a natural mosquito-parasite combination, the oocystaemias obtained in this study were unnaturally high. One likely explanation for these high oocystaemias is the high bird parasitaemia at the time of the blood feed [54]. To maximize infection success, most experimental mosquito infections, and the ones here were no exception, are carried out by feeding mosquitoes on hosts at the peak of their parasitaemia, a situation unlikely to be encountered by most blood feeding mosquitoes in the field. The large majority of birds in the field are survivors of past acute infections and have very low chronic parasitaemia [21,55-57]. Could the power to detect differences between insecticide resistant and susceptible mosquitoes have been increased had the mosquitoes had lower oocystaemias? This question could be resolved by feeding mosquitoes on chronically infected birds, which recent pilot studies have shown to render significantly lower infection rates and oocyst burdens (S. Cornet pers. com).

A marginal, albeit statistically significant, reduction of oocyst numbers in insecticide resistant mosquitoes (irrespective of the mechanism) was found in the third block of the wild-caught mosquito experiment. This result is 
intriguing as it goes in the expected direction of a reduction in parasite numbers associated with insecticide resistance [10-12]. The signal is however weak; on closer look, the effect only appears in two out of the three birds, and in only one of the blocks. Further experiments using chronic infections are needed before it can be determined whether this result was due to a statistical type I error (in other words, a false positive) or whether there is a real biological phenomenon underlying it.

\section{Conclusions}

Esterase and acetylcholinesterase-based insecticide resistance did not have a clear effect on the infection rate or oocyst burden in $C x$ pipiens mosquitoes, at least under the specific experimental conditions detailed above. Other key determinants of disease transmission, such as mosquito longevity and behaviour, or the parasite's incubation time within the mosquito, need to be investigated before concluding on the effects of these two mechanisms of insecticide resistance on the ability of these mosquitoes to transmit malaria [10]. The $C x$. pipiens - P. relictum system provides a good opportunity for investigating this question, not least because it is the only currently available non-human experimental model that uses a natural mosquito-Plasmodium combination and allows us to standardize the genetic background against which the insecticide resistant genes operate. The congruency of the results obtained between the isogenic strain and wild-caught mosquito experiments further suggests that, at least for the variables measured, the laboratory strains are not too far removed from field-caught mosquitoes, an additional advantage for experimental purposes.

The ultimate question is, however, whether insecticide resistance affects human malaria transmission, as this can have important public health consequences. The long and complex history of insecticide use in most endemic malarial areas greatly complicates the task of finding fully susceptible Anopheles individuals and therefore of making meaningful sympatric comparisons. The best current alternative is the establishment of isogenic lines of mosquitoes, though this option is lengthy to implement and not devoid of potential pitfalls $[10,13]$. Before these experimental challenges are overcome, research on animal malaria models remains a good alternative to understand the effect of insecticide resistance on the ability of mosquitoes to transmit malaria.

\section{Additional material}

Additional file 1: Description of statistical models used to analyse the influence of insecticide resistance on $C x$. pipiens infection.

\section{Acknowledgements}

The authors would like to thank Gabriele Sorci for providing us with the parasite isolates, Mylène Weill's group for the isogenic strain mosquitoes, Flore Zélé and Stéphane Cornet for their help with some of the preliminary experiments, and Philippe Perret for his invaluable help in all bird matters. Radiant Color NV kindly provided the mosquito pigments. JV is funded through an FCT grant attributed by the GABBA program, SG by an ANR Jeune Chercheur and an ERC Starting Grant, and AR by an ANR SEST grant.

\section{Author details}

'Génétique et Evolution des Maladies Infectieuses (UMR CNRS 2724), Centre de Recherche IRD, 911 Avenue Agropolis, 34394 Montpellier, France. ${ }^{2}$ Centre d'Ecologie Fonctionnelle et Evolutive (UMR CNRS 5175), 1919 Route de Mende, 34294 Montpellier, France.

\section{Authors' contributions}

$J V, A R$ and SG conceived and designed the experiments. JV, AR and AN performed the experiments. AN and JV carried out mosquitoes genotyping. $\mathrm{J}, \mathrm{AR}$ and SG wrote the paper. All authors read and approved the manuscript.

\section{Competing interests}

The authors declare that they have no competing interests.

Received: 6 October 2010 Accepted: 31 December 2010 Published: 31 December 2010

\section{References}

1. WHO: Global Malaria Control and Elimination: report of a technical review. Geneva 2008

2. Hemingway J, Ranson $\mathrm{H}$ : Insecticide resistance in insect vectors of human disease. Annu Rev Entomol 2000, 45:371-391.

3. Berticat C, Bonnet J, Duchon S, Agnew P, Weill M, Corbel V: Costs and benefits of multiple resistance to insecticides for Culex quinquefasciatus mosquitoes. BMC Evol Biol 2008, 8:104.

4. Berticat C, Boquien G, Raymond M, Chevillon C: Insecticide resistance genes induce a mating competition cost in Culex pipiens mosquitoes. Genet Res 2002, 79:41-47.

5. Sakyi KY, Sarfo B, Brown CA, Wilson MD, Boakye DA: Investigation into the fitness cost of kdr insecticide resistance in Anopheles gambiae malaria vectors. Am J Trop Med Hyg 2005, 73:155-155.

6. Bourguet D, Guillemaud T, Chevillon C, Raymond M: Fitness costs of insecticide resistance in natural breeding sites of the mosquito Culex pipiens. Evolution 2004, 58:128-135.

7. Read AF, Lynch PA, Thomas MB: How to make evolution-proof insecticides for malaria control. PLOS Biol 2009, 7(4):e100005.

8. Curtis CF, Miller JE, Hodjati MH, Kolaczinski JH, Kasumba I: Can anything be done to maintain the effectiveness of pyrethroid-impregnated bednets against malaria vectors? Philos Trans R Soc Lond B Biol Sci 1998, 353:1769-1775.

9. Lenormand T, Raymond M: Resistance management: the stable zone strategy. Proc R Soc Biol Sci Ser B 1998, 265:1985-1990.

10. Rivero A, Vezilier J, Weill M, Read AF, Gandon S: Insecticide control of vector-borne diseases: when is insecticide resistance a problem? PLOS Pathog 2010, 6(8):pii:e1001000.

11. McCarroll L, Hemingway J: Can insecticide resistance status affect parasite transmission in mosquitoes? Insect Biochem Mol Biol 2002, 32:1345-1351.

12. McCarroll L, Paton MG, Karunaratne S, Jayasuryia HTR, Kalpage KSP, Hemingway J: Insecticides and mosquito-borne disease. Nature 2000, 407:961-962

13. Curtis CF: Insecticide resistance and mosquito-borne disease. Lancet 2001, 357:656-656.

14. Enayati A, Hemingway J: Malaria management: past, present, and future. Annu Rev Entomol 2010, 55:569-591.

15. Pinto J, Lynd A, Elissa N, Donnelly MJ, Costa C, Gentile G, Caccone A, Do Rosario VE: Co-occurrence of East and West African kdr mutations suggests high levels of resistance to pyrethroid insecticides in Anopheles gambiae from Libreville, Gabon. Med Vet Entomol 2006, 20:27-32.

16. Dabire KR, Diabate A, Djogbenou L, Ouari A, N'Guessan R, Ouedraogo JB, Hougard JM, Chandre F, Baldet T: Dynamics of multiple insecticide 
resistance in the malaria vector Anopheles gambiae in a rice growing area in South-Western Burkina Faso. Malar J 2008, 7:188.

17. Corbel V, N'Guessan R, Brengues C, Chandre F, Djogbenou L, Martin T, Akogbeto M, Hougard JM, Rowland M: Multiple insecticide resistance mechanisms in Anopheles gambiae and Culex quinquefasciatus from Benin (West Africa) and operational challenge for malaria vector control. Am J Trop Med Hyg 2007, 77:230.

18. Vontas J, Blass C, Koutsos AC, David JP, Kafatos FC, Louis C, Hemingway J, Christophides GK, Ranson H: Gene expression in insecticide resistant and susceptible Anopheles gambiae strains constitutively or after insecticide exposure. Insect Mol Biol 2005, 14:509-521.

19. Okoye PN, Brooke BD, Hunt RH, Coetzee M: Relative developmental and reproductive fitness associated with pyrethroid resistance in the major southern African malaria vector, Anopheles funestus. Bull Entomol Res 2007, 97:599-605.

20. Vontas JG, McCarroll L, Karunaratne S, Louis C, Hurd H, Hemingway J: Does environmental stress affect insect-vectored parasite transmission? Physiol Entomol 2004, 29:210-213.

21. Valkiunas G, (Ed): Avian malaria parasites and other haemosporidia 2005.

22. Escalante AA, Ayala FJ: Phylogeny of the malarial genus Plasmodium, derived from ribosomal -RNA gene- sequences. Proc Natl Acad Sci USA 1994, 91:11373-11377.

23. Slater LB: Malarial birds: Modeling infectious human disease in animals. Bull Hist Med 2005, 79:261-294.

24. Weill M, Lutfalla G, Mogensen K, Chandre F, Berthomieu A, Berticat C, Pasteur N, Philips A, Fort P, Raymond M: Insecticide resistance in mosquito vectors. Nature 2003, 429:262-262.

25. Raymond M, Chevillon C, Guillemaud T, Lenormand T, Pasteur N: An overview of the evolution of overproduced esterases in the mosquito Culex pipiens. Philos Trans R Soc Lond B Biol Sci 1998, 353:1707-1711.

26. Lenormand T, Bourguet $D$, Guillemaud $T$, Raymond M: Tracking the evolution of insecticide resistance in the mosquito Culex pipiens. Nature 1999, 400:861-864

27. Rivero A, Magaud A, Nicot A, Vézilier J: Energetic costs of insecticide resistance in Culex pipiens mosquitoes. J Med Entomol 2010.

28. Moret $Y$, Schmid-Hempel P: Survival for immunity: the price of immune system activation for bumblebee workers. Science 2000, 290:1166-1168.

29. Atella GC, Bittencourt-Cunha PR, Nunes RD, Shahabuddin M, SilvaNeto MAC: The major insect lipoprotein is a lipid source to mosquito stages of malaria parasite. Acta Trop 2009, 109:159-162.

30. Ball GH, Chao J: Use of amino-acids by Plasmodium relictum oocysts invitro. Exp Parasitol 1976, 39:115-118.

31. Pasteur $N$, Nance $E$, Bons $N$ : Tissue localization of overproduced esterases in the mosquito Culex pipiens (Diptera: Culicidae). J Med Entomol 2001, 38:791-801.

32. Agnew P, Berticat C, Bedhomme S, Sidobre C, Michalakis Y: Parasitism increases and decreases the costs of insecticide resistance in mosquitoes. Evolution 2004, 58:579-586

33. Labbe P, Berticat C, Berthomieu A, Unal S, Bernard C, Weill M, Lenormand T: Forty years of erratic insecticide resistance evolution in the Mosquito Culex pipiens. PLoS Genet 2007, 3:2190-2199.

34. Tripet F: Ecological Immunology of mosquito-malaria interactions: of non-natural versus natural model systems and their inferences. Parasitology 2009, 136:1935-1942.

35. Berticat C, Dubois MP, Marquine M, Chevillon C, Raymond M: A molecular test to identify resistance alleles at the amplified esterase locus in the mosquito Culex pipiens. Pest Manag Sci 2000, 56:727-731.

36. Weill M, Malcolm C, Chandre F, Mogensen K, Berthomieu A, Marquine M, Raymond $\mathrm{M}$ : The unique mutation in ace-1 giving high insecticide resistance is easily detectable in mosquito vectors. Insect Mol Biol 2004, 13:1-7.

37. Rivero A, Ferguson HM: The energetic budget of Anopheles stephensi infected with Plasmodium chabaudi: is energy depletion a mechanism for virulence? Proc R Soc Biol Sci Ser B 2003, 270:1365-1371.

38. Service MW: Mosquito ecology: Field sampling methods. Second edition. London: Chapman \& Hall; 1993.

39. Alout $\mathrm{H}$, Berthomieu A, Hadjivassilis A, Weill M: A new amino-acid substitution in acetylcholinesterase 1 confers insecticide resistance to Culex pipiens mosquitoes from Cyprus. Insect Biochem Mol Biol 2007, 37:41-47.
40. Pasteur N, Pasteur G, Bonhomme F, Catalan J, Britton-Davidian J: Practical isozyme genetics New York: Halsted Press in Chichester; 1988.

41. Sinden RE, Dawes EJ, Alavi Y, Waldock J, Finney O, Mendoza J, Butcher GA Andrews L, Hill AV, Gilbert SC, Basanez MG: Progression of Plasmodium berghei through Anopheles stephensi is density-dependent. PLoS Pathog 2007, 3:2005-2016.

42. Vaughan JA: Population dynamics of Plasmodium sporogony. Trends Parasitol 2007, 23:63-70.

43. Briegel $\mathrm{H}$, Lea AO, Klowden MJ: Hemoglobinometry as a method for measuring blood meal sizes of mosquitos (Diptera, Culicidae). J Med Entomol 1979, 15:235-238.

44. Crawley MJ: The R Book John Wiley \& Sons, Ltd; 2007

45. Bolker BM: Ecological Models and Data in $R$ New Jersey: Princeton University Press; 2008.

46. Smith DL, McKenzie FE: Statics and dynamics of malaria infection in Anopheles mosquitoes. Malar I 2004, 3:13.

47. Dawes EJ, Churcher TS, Zhuang S, Sinden RE, Basanez MG: Anopheles mortality is both age- and Plasmodium-density dependent: implications for malaria transmission. Malar J 2009, 8.

48. Dong YM, Aguilar R, Xi ZY, Warr E, Mongin E, Dimopoulos G: Anopheles gambiae immune responses to human and rodent Plasmodium parasite species. PLoS Pathog 2006, 2:513-525.

49. Cohuet A, Osta MA, Morlais I, Awono-Ambene PH, Michel K, Simard F, Christophides GK, Fontenille D, Kafatos FC: Anopheles and Plasmodium: from laboratory models to natural systems in the field. EMBO Rep 2006, 7:1285-1289

50. Ferguson HM, Read AF: Why is the effect of malaria parasites on mosquito survival still unresolved? Trends Parasitol 2002, 18:256-261.

51. Tripet $F$, Aboagye-Antwi $F$, Hurd H: Ecological immunology of mosquitomalaria interactions. Trends Parasitol 2008, 24:219-227.

52. Boete C: Malaria parasites in mosquitoes: laboratory models, evolutionary temptation and the real world. Trends Parasitol 2005, 21:445-447.

53. Ferguson HM, Rivero A, Read AF: The influence of malaria parasite genetic diversity and anaemia on mosquito feeding and fecundity. Parasitology 2003, 127:9-19.

54. Taylor LH, Read AF: Determinants of transmission success of individual clones from mixed-clone infections of the rodent malaria parasite, Plasmodium chabaudi. Int J Parasitol 1998, 28:719-725.

55. Knowles SCL, Palinauskas V, Sheldon BC: Chronic malaria infections increase family inequalities and reduce parental fitness: experimental evidence from a wild bird population. J Evol Biol 2010, 23:557-569.

56. Bensch S, Waldenstrom J, Jonzen N, Westerdahl H, Hansson B, Sejberg D, Hasselquist D: Temporal dynamics and diversity of avian malaria parasites in a single host species. J Anim Ecol 2007, 76:112-122.

57. Kilpatrick AM, LaPointe DA, Atkinson CT, Woodworth BL, Lease JK, Reiter ME, Gross K: Effects of chronic avian malaria (Plasmodium relictum) infection on reproductive success of Hawaii Amakihi (Hemignathus virens). Auk 2006, 123:764-774.

doi:10.1186/1475-2875-9-379

Cite this article as: Vézilier et al.: Insecticide resistance and malaria transmission: infection rate and oocyst burden in Culex pipiens mosquitoes infected with Plasmodium relictum. Malaria Journal 2010 9:379.

\section{Submit your next manuscript to BioMed Central and take full advantage of:}

- Convenient online submission

- Thorough peer review

- No space constraints or color figure charges

- Immediate publication on acceptance

- Inclusion in PubMed, CAS, Scopus and Google Scholar

- Research which is freely available for redistribution

Submit your manuscript at www.biomedcentral.com/submit
C Biomed Central 